\title{
A study of the efficacy of a comprehensive memory enhancement program in healthy elderly persons
}

\author{
Richard C. Mohs ${ }^{\mathrm{a}, *}$, Teresa A. Ashman ${ }^{\mathrm{a}}$, Kathleen Jantzen ${ }^{\mathrm{a}}$, Marilyn Albert ${ }^{\mathrm{b}}$, \\ Jason Brandt ${ }^{\mathrm{c}}$, Barry Gordon ${ }^{\mathrm{c}}$, Xeno Rasmusson ${ }^{\mathrm{c}}$, Murray Grossman ${ }^{\mathrm{d}}$, \\ Diane Jacobs ${ }^{\mathrm{e}}$, Yaakov Stern ${ }^{\mathrm{e}}$ \\ ${ }^{a}$ Department of Psychiatry, Mount Sinai School of Medicine, New York, NY 10029, USA \\ ${ }^{\mathrm{b}}$ Harvard University School of Medicine, Boston, MA, USA \\ ${ }^{\mathrm{c}}$ Johns Hopkins University School of Medicine, Baltimore, MD, USA \\ ${ }^{\mathrm{d}}$ Department of Neurology, University of Pennsylvania School of Medicine, Philadelphia, PA, USA \\ ${ }^{\mathrm{e}}$ Columbia University College of Physicians and Surgeons, New York, NY, USA
}

Received 3 July 1997; revised 3 December 1997; accepted 19 December 1997

\begin{abstract}
Well educated, high functioning older adults (ages 60-90) were given a comprehensive memory enhancement training program to determine the effectiveness of the program in increasing cognitive performance and positively influencing self-assessments of memory efficacy. The 68 subjects who participated in the memory enhancement training were compared to 74 subjects who were enrolled in a video control group. Between subject differences were analyzed prior to the interventions and at three time points following the interventions (immediately post, 3 months and 6 months). Only one cognitive measure, assessing verbal memory, revealed a significant difference between the groups, with participants in the memory enhancement group showing less decline from baseline than the video control group immediately following the intervention but with no difference between groups at 6 months post-intervention. Several self-report measures showed that the memory enhancement training subjects experienced improved memory functioning and decreased memory concerns relative to the video control group. The self-reported effects of the training were sustained over the 6-month follow-up period. (c) 1998 Elsevier Science Ireland Ltd.
\end{abstract}

Keywords: Aging; Training program; Intervention; Adults; Efficacy

\footnotetext{
* Corresponding author, Psychiatry Service (116A), Mount Sinai School of Medicine, VA Medical Center, 130 W. Kingsbridge Road, Bronx, NY 10468, USA. Tel.: +1 7185849000 ext. 5176; fax: +1 718 9332121; e-mail: r_mohs@smtplink.mssm.edu
} 


\section{Introduction}

Because memory problems are common among elderly persons and are often a source of great concern, there is considerable interest in developing interventions that can improve memory in this population. Many studies have demonstrated that training memory skills will increase their use and that training on specific memory tasks can produce improvement on those tasks. As examples, focused training can improve the use of organization (Schmitt et al., 1981), use of imagery (Poon et al., 1980), use of the method of loci (Kliegl et al., 1989, 1990) and other mnemonic strategies in elderly adults (Yesavage, 1983; Yesavage et al., 1983; Verhaeghen et al., 1992).

A limitation of targeted training interventions has been the inability to generalize the benefits of training on a specific strategy beyond the tasks used for the training (Willis and Schaie, 1986). Two studies (Yesavage et al., 1983; Anschutz et al., 1987) did find significant improvement on memory tasks that differed from their method of loci training intervention. However, the majority of studies that have trained on one type of memory strategy and tested memory improvement on different types of tasks found either negligible transfer effects (Scogin and Bienias, 1988; Rebok and Balcerak, 1989) or limited effects (Stigsdotter-Neely and Backman, 1993a,b). In addition to lack of generalizability across memory tasks, specific training interventions have found that memory was improved immediately following the training sessions but not sustained at follow-up assessments (Yesavage, 1983; Scogin et al., 1985; Scogin and Bienias, 1988). Only a few studies have shown sustained improvement after 3 months (Dittmann-Kohli et al., 1991; Stigsdotter-Neely and Backman, 1993a,b) and 6 months (Sheikh et al., 1986; Stigsdotter-Neely and Backman, 1993a,b). There is also some question about whether targeted interventions improve memory over and above the improvements which occur as a result of general social stimulation. Schaeffer and Poon (1982) found that older adults improved memory performance through social support groups without any memory training. Their results suggested that training on specific memory skills may not be the only factor enhancing memory performance, but that social stimulation alone increases cognitive stimulation and, in turn, improves memory.

Recent studies have moved beyond tests of the effects of memory training strategies and have investigated the effects of more comprehensive interventions that include several cognitive and non-cognitive techniques (Dittmann-Kohli et al., 1991; Verhaeghen et al., 1992; Stigsdotter-Neely and Backman, 1993a,b, 1995). If older subjects receive training on mnemonic techniques only, there is generally no difference in memory performance between the training and the control groups when examined after 3 years (Anschutz et al., 1987; Scogin and Bienias, 1988). Yet when a multifactorial intervention that combined different memory enhancing techniques and non-cognitive (e.g. relaxation) skills was employed, improvement in memory performance was found (Yesavage, 1984) and was sustained for 6 months (Stigsdotter-Neely and Backman, 1993a) and up to 3.5 years (Stigsdotter-Neely and Backman, 1993b) following training.

The results of Stigsdotter-Neely and Backman (1993a,b) studies demonstrated an effect of multifactorial training that included cognitive (encoding and imagery) and non-cognitive (relaxation techniques) domains. The meta-analysis of Verhaeghen et al. (1992) also suggested that treatment gains in memory increase when subjects' are given extensive pre-training in either cognitive or non-cognitive areas. However, the extent to which these interventions contribute to participants' memory performance outside of the testing environment remains uncertain. Only 39\% of Stigsdotter-Neely and Backman (1993b) subjects stated that they used the mnemonic techniques 6 months after training.

There is evidence that one important aspect of a cognitive training program for older persons is the extent to which it enhances their sense of 'self-efficacy' regarding memory performance. The term self-efficacy was conceptualized by Bandura (1977) and has been defined as one's sense of competence and confidence related to a specific performance in a given domain. While supportive feedback following cognitive training has been 
shown to enhance self-efficacy in elderly subjects, Lachman et al. (1992) did not find any differences in memory performance between treatment and control groups. Dittmann-Kohli et al. (1991) found a difference between training and control groups on subjective appraisals of their memory performance, but no differences between groups were found on the objective measures of memory. Lachman et al. (1987) found that older adults can increase the accuracy of their memory predictions when they have pre-exposure to the task being tested. While Stigsdotter-Neely and Backman (1993a,b, 1995) studies addressed the issues of generalizability by using several memory tasks and memory relevant factors of attention and concentration along with relaxation techniques, they did not include self-report measures.

The present study was designed to evaluate the effectiveness of a 9-week memory enhancement course in which elderly persons received instruction and practice in a wide variety of techniques and strategies for dealing with memory problems. The program was not designed to train subjects on any one cognitive task, since much of the previous research has suggested that specifically targeted training does not translate to other cognitive domains. As indicated, memory performance can be improved most successfully and more durably when training incorporates a multifactorial approach (Stigsdotter-Neely and Backman, 1993a,b). Improvements on memory task performance can also be provided through supportive feedback during training which enhances self-efficacy (Lachman et al., 1987, 1992; Dittmann-Kohli et al., 1991). Finally the subjective experiences of negative mood (West et al., 1984) and memory complaints (Gilewski et al., 1990) can contribute to decreased memory performance. To date no study has combined a multifactorial training approach with supportive feedback and measurements of self-efficacy, memory complaints and mood. The current study investigated the utility of a comprehensive memory training program with older adults that was distinctive from previous studies by including a broad array of cognitive and non-cognitive tasks including self-report outcome measures.

\section{Method}

\subsection{Participants}

One hundred forty-two subjects (110 women and 32 men) were recruited at five sites participating in the Charles A. Dana Foundation Consortium on Memory Loss and Aging. The sites were clinics and residential facilities or community centers for seniors affiliated with Johns Hopkins University School of Medicine, Harvard University School of Medicine, University of Pennsylvania, Columbia University College of Physicians and Surgeons, or Mount Sinai School of Medicine. At four of the sites, subjects were recruited from residential facilities or community centers for seniors, while at the fifth site, Mount Sinai, participants were recruited from the community through advertisements. The inclusion criteria for the study were: subjects had to be 60 years or older, have at least a tenth grade education and have a score above the 25th percentile on the Mini Mental State Exam relative to age and education (MMSE: Folstein et al., 1975; Crum et al., 1993). Subjects were excluded if they had a diagnosis of Alzheimer's Disease or any dementing illness, were taking any medications affecting cognitive functioning, had a history of head trauma with loss of consciousness, or had a history of any neurological, any AXIS I psychiatric disorder (DSM-IV, 1995), or substance abuse disorder in the past 10 years. Once subjects were recruited and met inclusion and exclusion criteria, they were randomly assigned at each site to either the memory training group or a video tape educational control group, with the constraint that the two groups at each site were matched on age, sex, and educational level. Numbers of participants varied by site from a low of 17 (nine video, eight memory enhancement) to a high of 34 (19 video, 15 memory enhancement). Subject characteristics are presented in Table 1. ANOVAs conducted on subject characteristics (age, sex, educational level, IQ and MMSE) revealed no differences between the two groups $(F \mathrm{~s}<1)$. There also was no significant difference on subject characteristics between sites $(F \mathrm{~s}<1)$. The mean age of the total 
Table 1

Subjects' characteristics

\begin{tabular}{lcc}
\hline Variable & Group & \\
\cline { 2 - 3 } & $\begin{array}{l}\text { Memory } \\
n=68\end{array}$ & $\begin{array}{c}\text { Video } \\
n=74\end{array}$ \\
& $53 / 15$ & $57 / 17$ \\
Female/male & & \\
Age & 78.3 & 78.8 \\
Mean & 7.4 & 6.5 \\
S.D. & & \\
Years of education & 16.0 & 15.7 \\
Mean & 2.7 & 2.5 \\
S.D. & & \\
Mini-Mental Status Exam & 28.9 & 28.8 \\
Mean & 1.2 & 1.4 \\
S.D. & & \\
Estimated IQ & 128.2 & 126.8 \\
Mean & 18.0 & 16.5 \\
S.D. &
\end{tabular}

sample was 78.6 years (range 60-94, S.D. $=6.92$ ). The mean educational level was 15.9 years (range 10-20, S.D. $=2.55$ ). Subjects' IQ scores were estimated from selected subtests (Information, Vocabulary and Similarities) of the Wechsler Adult Intelligence Scale-Revised (WAIS-R: Satz and Mogel, 1953; Wechsler, 1981). The average estimated IQ level for all subjects was almost two standard deviations above the general population $($ mean $=127.5$, S.D. $=17.19)$, with no site having an average less than one standard deviation above the mean. All subjects gave written informed consent before participating in the study.

\subsection{Outcome measures}

The complete test battery was administered five times: two baseline assessments and three postintervention assessments (immediate, 3 months and 6 months). The battery included four objective cognitive measures and several subjective self-report measures. The cognitive measures were selected to assess two types of memory (verbal and non-verbal) and cognitive abilities other than memory which have been shown to decline with age (verbal fluency and object naming).

\subsubsection{Neuropsychological measures}

Word lists from the California Verbal Learning Test (CVLT: Delis et al., 1986, 1991) assessed verbal memory by the recall of concrete words. Subjects were presented with a list of 16 words that were read aloud at a rate of $5 \mathrm{~s}$ per word. Subjects were asked to recall as many words as possible at the end of the list presentation. The list was repeated across five acquisition trials. The combined total of words learned throughout the five trials was used in all analyses.

The Biber Figure Learning Test (BFLT: Glosser et al., 1989) is a non-verbal, visuospatial analogue to the CVLT, testing for the recall of abstract figures. Similarly for the CVLT, subjects were presented with 15 abstract figures across five learning trials. At the end of each trial, subjects were asked to recall as many figures as possible. Again, we analyzed only the cumulative learning score for the five exposures.

Verbal fluency was measured by the Verbal Fluency Test (VF: Rosen, 1980). Subjects spontaneously generated words for $1 \mathrm{~min}$ in each of three categories. The categories differed at each session visit (e.g. vegetables, fruit, flowers, animals), with only the first baseline assessment and the 6-month follow-up assessment using the same three categories. Reproductions were scored by a single rater who was blind to treatment condition. A total score was given for the total number of words generated in all three categories.

A subset of items from the Boston Naming Test (BNT: Kaplan et al., 1983) was used to assess object naming ability. The subset was taken from the neuropsychological battery of the Consortium to Establish a Registry for Alzheimer's Disease (CERAD: Morris et al., 1989). The 15 items were selected so that five each were high, medium and low in frequency. In all cases the neuropsychological tests were given in the same sequence for the five session visits, although equivalent forms of the CVLT, BFLT and VF were given to both groups at different sessions.

\subsubsection{Subjective measures}

Self-report measures were given to assess the subjects' beliefs about their memory functioning 
and their reported memory difficulties. The Memory Controllability Inventory (MCI: Lachman et al., 1995) was used to assess the self-efficacy of memory functioning. The MCI is a 20 -item questionnaire with the following subscales: aging concerns, perceived memory ability and perceived memory control. The Memory Functioning Questionnaire (MFQ: Gilewski et al., 1990) is a 63-item questionnaire which was used to evaluate memory complaints and abilities in the areas of mnemonic usage, seriousness of memory problems, retrospective memory functioning and total forgetting. The total forgetting scale was an average score of three forgetting subscales (frequency of forgetting, frequency of forgetting during reading and remembering past events). The participants' subjective levels of anxiety and depression were assessed with the Profile of Moods Scale (POMS: Spielberger, 1971).

\subsection{Procedure}

All potential subjects received an initial screening which consisted of the MMSE, sociodemographic information, and psychiatric and medical histories. Once the screening was completed, the study consisted of two baseline pre-testings followed by the intervention (memory enhancement training or video tape educational control group) and three post-testings. Two baseline pre-testings were incorporated into the study design to obtain an accurate baseline level for each subject. While there was a significant difference between the first baseline and the second, the difference was the same for both groups. The baselines were combined in an effort to reduce the impact of practice effects that occurred with the second baseline testing and control for regression toward the mean. The first post-testing immediately followed the 9-week intervention, the second occurred 3 months later and the third after 6 months of training. The baseline and three subsequent follow-up assessments included all measures of cognitive and non-cognitive functioning. The psychometricians who conducted the neuropsychological battery were trained in all assessment procedures and were blind to the subjects' group assignment. The interventions were conducted in two groups at each of the five sites. Each group met for 9 weeks with each session lasting 90 min. Both groups were led by a trained facilitator.

\subsection{Memory training}

The memory enhancement training group received nine training sessions, each lasting $90 \mathrm{~min}$. The first session was an overview of the nature and common misperceptions about memory along with an outline of topics to be presented in the remaining sessions. Subjects were invited to ask questions and describe their specific memory complaints so that the facilitator could use some identifiable examples in the following sessions. Following the initial overview session, the remaining eight sessions had a consistent structure to facilitate an effective presentation (Table 2). Each session began with a review and a simple quiz on the content of the previous session and homework assignments. The facilitator would then present the current session's didactic information (Table 3). Once the new information was given, subjects were encouraged to describe personal experiences within the context of the session's lecture. The facilitator would incorporate these examples to demonstrate how to use the lecture's principles to solve the subjects' everyday memory problems. After the discussion, subjects were given a set of exercises that could be performed easily to enhance self-efficacy. At the end of the session, the facilitator would give subjects feedback and homework for the following week.

A complete list of topics covered in the nine sessions of the memory training group is presented in Table 2. As the facilitator outlined the structure of the course in the first session, she or

Table 2

Basic structure of a memory enhancement training session

- Review and quiz on previous session's material

- Presentation of new didactic information

- Subjects give personal examples of memory problems

- Facilitator relates concrete exercises to address the problems

- Positive feedback

- Homework assignment 
Table 3

Memory training curriculum

Session 1: How Your Memory Works

Session 2: Stages in the Processes of Remembering

Session 3: Making the Most of Your Memory

Session 4: Attention and Concentration

Session 5: Making Remembering Easier

Session 6: Practice Makes Perfect

Session 7: Remembering Text Information

Session 8: Remembering Names and Faces

Session 9: Maintaining Memory Improvements

he emphasized that the goal was to learn strategies to improve memory. The subjects were taught the five basic principles to enhance memory that would be used throughout the training course: meaningfulness, organization, visualization, association and attention. Session 2 focused on the three stages of memory as a means of clarifying the difficulty of remembering new vs. old learning. Subjects were instructed to learn new information (i.e. a phone number) to emphasize the $7 \pm 2$ rule of short-term memory. Session 3 combined information on factors that can impair (i.e. alcohol and drugs) and enhance memory (i.e. rest, good health, planning) with specific ideas on how one rid oneself of stress and anxiety. Part of the session was devoted to training subjects on the use of the 'Jacobson Relaxation and Systematic Desensitization Technique' (Jacobson, 1938). Techniques for improving attention and concentration were demonstrated in session 4. Three principal elements of attention were highlighted: alertness, selectivity and motivation. In sessions 5 and 6 the subjects were trained and given practice time on specific techniques like external memory aids (e.g. appointment books, diaries, use of a 'memory spot' for items often misplaced) and ways to remember word lists through categorization and chunking. Sessions 7 and 8 emphasized remembering text, names and faces. Subjects were taught to organize text information according to themes that were meaningful to them. Techniques for remembering names and faces included repeating the names throughout the conversation and visualizing either the name or the face with a concrete object to facilitate recall.
The final session reviewed all techniques learned and suggested ways to maintain these skills along with a positive attitude about memory. Further details about the memory enhancement program can be obtained from the corresponding author.

\subsection{Video training}

The video control group met over the same period of time as the memory enhancement group to provide a comparable time of exposure. Instead of lectures and training, their sessions were devoted to watching and discussing videos related to cognitive functioning chosen from the PBS series 'The Brain' and 'The Mind' (Table 4). There was some discussion of memory problems that included questions and answers. However, no specific memory skills or problem solving techniques were taught and there was no discussion of specific memory difficulties experienced by the participants.

\section{Results}

\subsection{Data analyses}

Subjects' mean scores for each measure were submitted to separate mixed-model Analyses of Covariance (ANCOVAs) using factors of Group, Site and Session Visit, with repeated measures on the last factor. The design was two groups (memory enhancement and video control) by six sites (two sites at Johns Hopkins University) by post-

Table 4

Video tape training series

Session 1: The Enlightened Machine ${ }^{\mathrm{a}}$

Session 2: Stress and Emotion ${ }^{\mathrm{a}}$

Session 3: Learning and Memory ${ }^{a}$

Session 4: Madness ${ }^{\mathrm{a}}$

Session 5: States of Mind ${ }^{\mathrm{a}}$

Session 6: Aging ${ }^{\mathrm{b}}$

Session 7: Language ${ }^{\mathrm{b}}$

Session 8: Thinking

Session 9: Review and Discussion

\footnotetext{
${ }^{\mathrm{a}}$ The Brain.

${ }^{\mathrm{b}}$ The Mind.
} 
intervention session (immediate, 3 months and 6 months). The covariates included age, years of education, gender, MMSE score, estimated IQ and baseline scores. Because there were a few subjects with missing data at one or more post-intervention test sessions, the data were analyzed in two ways. The primary, or fully evaluable, patient analysis used subjects with data at all visits; a secondary analysis was done with the last observation carried forward (LOCF) method, in which data from the last visit were substituted for missing values at later visits. In all instances but one the two analyses gave similar results, and for the one discrepant case we based conclusions on the more conservative result.

The principal objective was to evaluate the differences between the effect of the two treatment interventions. The comparisons of interest were the main effect for Group and the interactions involving Groups. The dependent variables were four measures of memory performance (CVLT, BFLT, BNT and VF) and three self-report questionnaires (MCI, MFQ and POMS). The $t$-tests between the first and the second baseline scores showed a significant difference suggesting that a possible learning effect occurred in the week's interval between the two sessions. However, since it was not possible to predict if such a learning effect would remain for the first post-intervention session, the average of the two baseline sessions was used as the baseline score to account for any regression toward the mean.

Partial correlation coefficients were computed between the scores on the performance measures and the self-report measures at each post-intervention assessment. The correlations were to determine if there was any significant relationship between the subjects' subjective experience of memory problems and their actual objective memory performances controlling the effect of the baseline scores.

\subsection{Treatment group differences on objective performance measures}

The means and standard deviations for each of the objective performance measures (CVLT, BFLT, VF and BNT) are presented in Table 5.
Table 5

Means and standard deviations for cognitive measures by treatment group and time

\begin{tabular}{lllll}
\hline & Baseline & Immediate & 3 Months & 6 Months \\
\hline CVLT $^{\mathrm{a}}$ & & & & \\
Memory & $47.6 \pm 10.44$ & $46.9 \pm 12.1$ & $47.7 \pm 12.7$ & $48.0 \pm 12.2$ \\
& $(n=66)$ & $(n=64)$ & $(n=60)$ & $(n=58)$ \\
Video & $47.1 \pm 10.6$ & $44.9 \pm 12.5$ & $45.0 \pm 11.6$ & $48.5 \pm 12.4$ \\
& $(n=74)$ & $(n=69)$ & $(n=66)$ & $(n=66)$
\end{tabular}

BFLT

$$
\begin{array}{lllll}
\text { Memory } & 117.3 \pm 35.1 & 117.6 \pm 38.5 & 121.8 \pm 40.4 & 122.0 \pm 36.5 \\
& (n=65) & (n=63) & (n=60) & (n=55) \\
\text { Video } & 112.3 \pm 37.1 & 113.3 \pm 40.3 & 116.5 \pm 40.8 & 114.3 \pm 40.3 \\
& (n=72) & (n=67) & (n=66) & (n=65)
\end{array}
$$

VF

$$
\begin{array}{ccccc}
\text { Memory } & 47.1 \pm 12.3 & 34.3 \pm 8.4 & 51.0 \pm 14.2 & 48.7 \pm 11.0 \\
& (n=67) & (n=64) & (n=60) & (n=58) \\
\text { Video } & 47.9 \pm 10.4 & 34.6 \pm 8.8 & 51.1 \pm 12.4 & 49.8 \pm 12.6 \\
& (n=74) & (n=69) & (n=66) & (n=66)
\end{array}
$$

$$
\begin{array}{ccccc}
\begin{array}{c}
\text { BNT } \\
\text { Memory }
\end{array} & 14.3 \pm 1.0 & 14.6 \pm 1.1 & 14.5 \pm 0.75 & 14.4 \pm 0.87 \\
& (n=65) & (n=64) & (n=60) & (n=57) \\
\text { Video } & 14.3 \pm 1.2 & 14.5 \pm 1.2 & 14.6 \pm 0.82 & 14.6 \pm 0.75 \\
& (n=72) & (n=68) & (n=66) & (n=66)
\end{array}
$$

${ }^{\mathrm{a}}$ Group $\times$ Session Visit interaction: $F_{2,212}=3.54, P<0.04$.

The only measure of memory for which there was a significant treatment effect on the repeated measures ANCOVAs was the total (trials 1-5) score on the CVLT and this difference was in the form of an interaction effect of Group $\times$ Session Visit $\left(F_{2,214}=3.54, P<0.03\right)$. None of the other measures revealed a significant treatment effect. Fig. 1 shows the mean baseline and post-intervention CVLT scores, indicating that the memory enhancement group performed better than the video training group at the immediate and 3month assessment times, though the difference did not hold at 6 months.

\subsection{Treatment effects for the subjective measures}

Table 6 presents the means and standard deviations on the MFQ. Analyses of all four subscales used age, years of education, gender, MMSE 


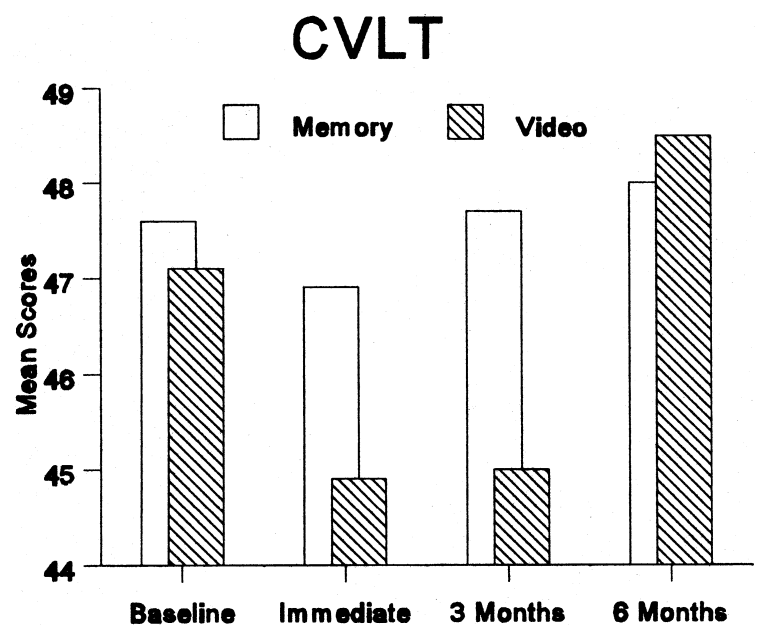

Fig. 1. CVLT baseline and post-intervention scores.

score, estimated IQ and the baseline scores as the covariates. On the MFQ mnemonic usage scale there was a main effect for Group $\left(F_{1,99}=9.26\right.$, $P<0.004)$, indicating that the memory training group reportedly used mnemonics more frequently than the video training group immediately following the intervention through the 6month follow-up. Fig. 2 demonstrates the substantial difference between groups. The fully evaluable patient analysis showed a marginally significant interaction of Group $\times$ Session Visit for total forgetting $\left(F_{2,178}=4.02, P<0.05\right)$, but this result was not confirmed by the LOCF analysis $\left(F_{2,260}<1, P>0.10\right)$.

The means and standard deviations for the three subscales of the MCI are listed in Table 7. ANCOVAs revealed main effects for Group on aging concern questions $\left(F_{1,99}=9.91, P<0.003\right)$ and perceived ability of memory functioning $\left(F_{1,96}\right.$ $=20.54, P<0.001)$. Figs. 3 and 4 depict the improvement in self-efficacy in the memory training group over the video group after the intervention. There was also a main effect for Session Visit on aging concerns $\left(F_{2,200}=3.09, P<0.05\right)$, suggesting that all subjects had fewer aging concerns post-intervention. Again, age, years of education, gender, MMSE scores, estimated IQ and baseline scores were covaried for all three subscales $(P<$ 0.001). The repeated measures ANCOVAs for the POMS did not reveal any significant effects for either reported anxiety or depression.

\subsection{Objective vs. subjective measures and individual differences}

Partial correlation coefficients were used to determine if there was a significant relationship between subjects' subjective experience of me-

Table 6

Means and standard deviations for the Memory Functioning Questionnaire by treatment group and time of assessment

\begin{tabular}{|c|c|c|c|c|}
\hline MFQ & Baseline & Immediate & 3 Months & 6 Months \\
\hline \multicolumn{5}{|c|}{ Mnemonic usage $^{\mathrm{a}}$} \\
\hline Memory & $2.6 \pm 1.1$ & $2.1 \pm 0.81$ & $2.3 \pm 1.1$ & $2.2 \pm 0.91$ \\
\hline Video & $2.8 \pm 1.1$ & $2.8 \pm 1.4$ & $2.7 \pm 1.4$ & $2.7 \pm 1.0$ \\
\hline \multicolumn{5}{|c|}{ Seriousness } \\
\hline Memory & $4.8 \pm 1.2$ & $4.6 \pm 1.3$ & $4.8 \pm 1.3$ & $4.7 \pm 1.3$ \\
\hline Video & $4.6 \pm 1.0$ & $4.7 \pm 1.1$ & $4.6 \pm 1.1$ & $4.6 \pm 1.2$ \\
\hline \multicolumn{5}{|c|}{ Retrospective functioning } \\
\hline Memory & $3.3 \pm 1.1$ & $3.2 \pm 1.2$ & $3.3 \pm 0.90$ & $3.3 \pm 1.0$ \\
\hline Video & $3.1 \pm 1.1$ & $3.2 \pm 1.2$ & $3.0 \pm 1.1$ & $3.1 \pm 1.2$ \\
\hline \multicolumn{5}{|c|}{ Total forgetting } \\
\hline Memory & $4.2 \pm 0.78$ & $4.4 \pm 0.84$ & $4.4 \pm 0.78$ & $4.5 \pm 0.76$ \\
\hline Video & $4.2 \pm 0.68$ & $4.4 \pm 0.67$ & $4.4 \pm 0.79$ & $4.4 \pm 0.74$ \\
\hline
\end{tabular}

\footnotetext{
${ }^{\text {a }}$ Group main effect: $F_{1,99}=9.26, P<0.01$; lower score indicates more usage.
} 


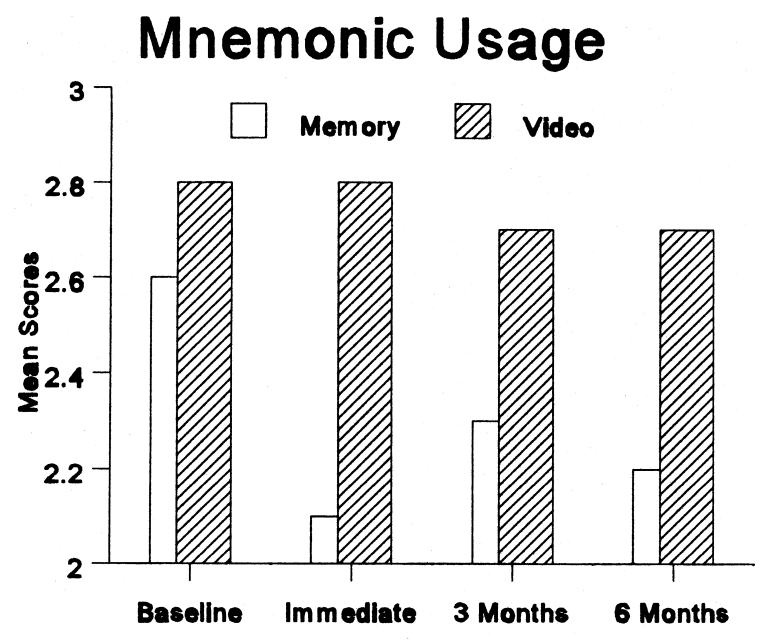

Note: Lower scores indicate more usage

Fig. 2. Mnemonic usage (MFQ) baseline and post-intervention scores. Note: lower scores indicate more usage.

Table 7

Means and standard deviations for the Memory Controllability Inventory by treatment group and time of assessment

\begin{tabular}{|c|c|c|c|c|}
\hline MCI & Baseline & Immediate & 3 Months & 6 Months \\
\hline \multicolumn{5}{|c|}{ Perceived ability $^{\mathrm{a}}$} \\
\hline Memory & $\begin{array}{l}5.0 \pm 1.0 \\
(n=65)\end{array}$ & $\begin{array}{l}5.5 \pm 0.93 \\
(n=64)\end{array}$ & $\begin{array}{l}5.4 \pm 0.94 \\
(n=60)\end{array}$ & $\begin{array}{l}5.5 \pm 1.0 \\
(n=58)\end{array}$ \\
\hline Video & $\begin{array}{l}4.9 \pm 0.82 \\
(n=68)\end{array}$ & $\begin{array}{l}4.9 \pm 0.93 \\
(n=68)\end{array}$ & $\begin{array}{l}4.9 \pm 0.97 \\
(n=66)\end{array}$ & $\begin{array}{l}4.8 \pm 1.0 \\
(n=66)\end{array}$ \\
\hline \multicolumn{5}{|c|}{ Perceived control } \\
\hline Memory & $\begin{array}{l}4.4 \pm 0.54 \\
(n=64)\end{array}$ & $\begin{array}{l}4.2 \pm 0.74 \\
(n=64)\end{array}$ & $\begin{array}{l}4.4 \pm 0.73 \\
(n=60)\end{array}$ & $\begin{array}{l}4.2 \pm 0.69 \\
(n=58)\end{array}$ \\
\hline Video & $\begin{array}{l}4.3 \pm 0.58 \\
(n=69)\end{array}$ & $\begin{array}{l}4.4 \pm 0.61 \\
(n=68)\end{array}$ & $\begin{array}{l}4.4 \pm 0.65 \\
(n=65)\end{array}$ & $\begin{array}{l}4.3 \pm 0.66 \\
(n=66)\end{array}$ \\
\hline \multicolumn{5}{|c|}{ Aging concerns ${ }^{\mathrm{b}}$} \\
\hline Memory & $\begin{array}{l}2.9 \pm 0.89 \\
(n=67)\end{array}$ & $\begin{array}{l}2.7 \pm 1.1 \\
(n=64)\end{array}$ & $\begin{array}{l}2.7 \pm 1.1 \\
(n=60)\end{array}$ & $\begin{array}{l}2.8 \pm 0.94 \\
(n=58)\end{array}$ \\
\hline Video & $\begin{array}{l}3.1 \pm 0.88 \\
(n=68)\end{array}$ & $\begin{array}{l}3.1 \pm 1.0 \\
(n=67)\end{array}$ & $\begin{array}{l}3.1 \pm 1.0 \\
(n=66)\end{array}$ & $\begin{array}{l}3.1 \pm 0.94 \\
(n=66)\end{array}$ \\
\hline
\end{tabular}

a Group main effect: $F_{1,96}=20.54, P<0.001$.

${ }^{\mathrm{b}}$ Group main effect: $F_{1,99}=9.91, P<0.01$; lower score indicates less concern.

mory problems and improvements and their objective memory performances. No significant cor-

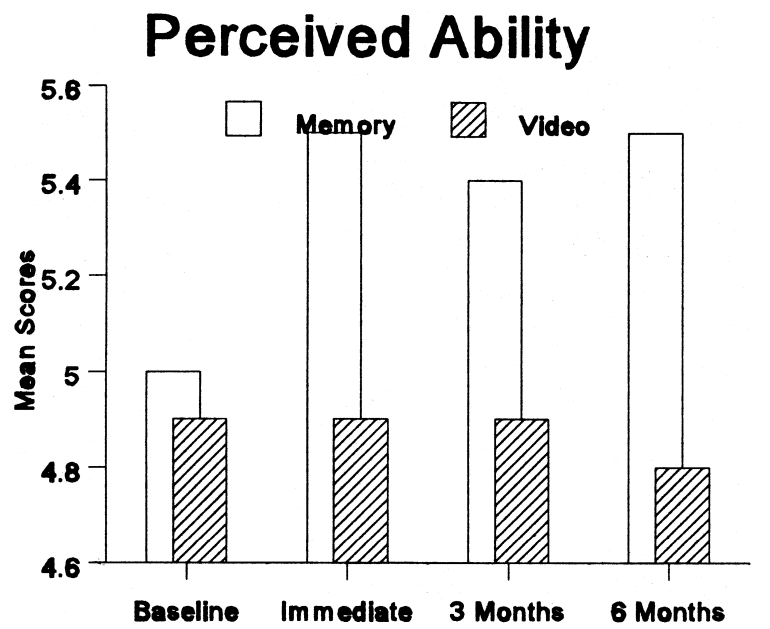

Fig. 3. Perceived ability (MCI) baseline and post-intervention scores.

relations were found between the objective and subjective measures at any post-intervention session visit controlling for the baseline scores. None of the demographic variables (age, education, gender) were significant predictors of change on the CVLT or subjective measures.

\section{Discussion}

The results from this comprehensive memory study, designed to compare a memory enhancement intervention with a general educational video training program, revealed differences between groups on verbal memory and sustained improvement in the memory training group's subjective experience of memory problems. Of the four cognitive tasks given at each assessment time, only verbal memory assessed by the CVLT showed a significant difference between groups. This finding was significant, but not overly robust, since the difference in performance between groups was fairly small and was largely due to the fact that the performance of the video group declined from baseline. Thus, the memory enhancement program did lead to a transient difference in verbal memory performance for the memory enhancement group relative to the video control group, but it is not clear to what extent partici- 


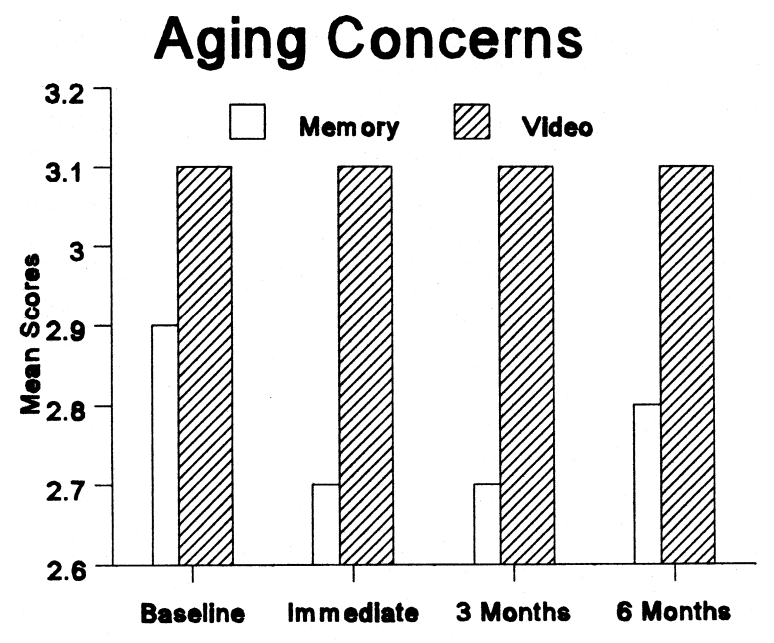

Note: Lower scores indicate fewer concerns

Fig. 4. Aging concerns (MCI) baseline and post-intervention scores. Note: lower scores indicate fewer concerns.

pants improved over their own baseline. None of the other cognitive measures of non-verbal ability, object naming or verbal fluency reflected any significant differences between the two groups, nor revealed any positive increase in performance.

More robust and possibly more important differences were found between the two groups on the self-report measures. Those subjects who received extensive training and feedback reported higher levels of self-efficacy on measures such as perceived ability of their memory functioning and fewer aging concerns related to memory. The memory enhancement group also indicated that they used mnemonics more frequently than the video group. This finding suggests that the memory training subjects continued to use techniques learned in the course for up to 6 months after completion. The lack of significant differences on the subjective mood ratings was not surprising. All subjects reported a relatively low level of anxiety or depression at any assessment time. The low variability on these mood measures reflects the general well-being of all participants. The lack of correlation between mood ratings and performance scores on subjective measures of memory complaints and self-efficacy supports previous findings among healthy elderly subjects (Schaeffer and Poon, 1982; West et al., 1984).

Although the study was conducted at several different locations, there were no significant differences among subjects at any site. Site was included as a factor in the analyses to check for the generalizability of the results. No interaction was found between Site and Group nor Site and Session Visit, thus indicating that the subjects responded similarly regardless of location.

The present results demonstrate that a comprehensive memory enhancement program can have beneficial effects if provided to highly motivated older persons. Those benefits were reflected almost entirely in participants' improved ratings of memory self-efficacy, increased use of mnemonics and decreased concern about memory problems associated with aging. Because there was an active control condition, these sustained improvements in reported self-efficacy and the short-lived improvement in verbal memory cannot be attributed to social stimulation (Schaeffer and Poon, 1982). Rather they must be attributed to the combination of education about memory, training in relaxation and memory enhancement techniques and feedback incorporated into the memory enhancement program.

While there were clear-cut benefits associated with the memory enhancement program, the results of this study raise a number of important questions. Firstly, how to design better criteria for determining the efficacy of such a program? In contrast to the number of older studies that used only neuropsychological test scores to determine efficacy (Verhaeghen et al., 1992; StigsdotterNeely and Backman, 1993a,b, 1995), we used both cognitive tests and self-report measures. Our findings show a discrepancy between increased efficacy and improvement in cognitive performance as assessed by our neuropsychological measures. Nevertheless, the training may have had real value in subjects' day to day functioning. The training emphasized how to decrease the level of forgetting on these everyday tasks. However, the cognitive measures used did not tap the relevant everyday concerns of the subjects; thus the subjective experience of improvement may be 
based on everyday experiences with memory in non-laboratory tasks. Also in terms of total forgetting, the memory training group did not report significant decreases in forgetting. Instead they reported that they had more potential ability to remember than they believed prior to enrollment in the study. Future studies may need to incorporate additional measures of real-world memory performance in an effort to provide evidence of the impact of the memory enhancement program on participants' lives.

The present results also raise some concern over the time period needed for an effective intervention and the need for a long-term followup. Memory problems and concerns in the elderly develop over many years, and it is unlikely that such problems ever totally resolve. It is quite possible that the effective use of strategies and techniques for dealing with memory problems may continue to develop over long periods of time if support and guidance are provided. Informal feedback from participants in the memory enhancement group indicated that they believed ongoing 'booster' sessions conducted at approximately monthly intervals would have further enhanced the utility of the program. Future research clearly needs to investigate the efficacy of these programs over time periods that are relevant to older persons (Stigsdotter-Neely and Backman, 1993a).

Lastly, the selection of participants and control procedures for studies such as this one must be considered carefully. All subjects freely volunteered to participate and their sociodemographic information and general eagerness to be a part of the study point to their overall high level of functioning. A large percentage went to college, which was not that common at the time that they would have attended. The high functioning level of the subjects at baseline could have yielded ceiling effects and the high estimated IQ of the sample (close to two standard deviations above the norm for their peers in the general population) limits generalizability of the results. It is quite possible that a training program such as the one used in this study could produce more improvement on standard measures of memory in a population of elderly persons with poorer base- line performance. In addition, choice of the control condition also could have diminished the possible benefits of the memory enhancement program. The video group may have been cognitively stimulated by the interaction with their peers (Schaeffer and Poon, 1982) and they were given information on the brain with some allowance made for a discussion of memory related issues.

Collectively, these results suggest that among high functioning older persons, an educational program targeting specific memory complaints, providing substantive feedback and support in addressing such complaints can enhance individual subjective experience of memory abilities and, possibly, some memory performance. Self-efficacy beliefs were not correlated with memory performance on any of the cognitive domains assessed. The positive increase in self-efficacy and the decrease in memory complaints following the memory enhancement training highlight the distinction between objective and subjective memory assessments. To further ascertain whether improvement in self-efficacy leads to actual improvement in memory tasks outside of the laboratory, future research in this area could include cognitive and other measures that have more real world relevance. The long-term effect of such interventions should be examined.

\section{Acknowledgements}

This research was supported by a grant from the Charles A. Dana Foundation. The following individuals provided helpful input into the development, implementation and structuring of the study protocol: Mohsen Aryan, Ph.D., Mount Sinai Medical Center; Richard Azeuta, M.A., Mount Sinai Medical Center; Kit Bick, Ph.D., Charles A. Dana Foundation; Felicita Cabrera, Mount Sinai Medical Center; Kit Carsen, M.S., Johns Hopkins University School of Medicine; Kelly Culbert, M.S., E.D., University of Pennsylvania; Charlie Hall, M.S., Johns Hopkins University School of Medicine; Phillip Harvey, Ph.D., Mount Sinai Medical Center; Jennifer Kwass, University of Pennsylvania; Robert Marcario, Johns Hopkins University School of Medicine; 
Jim O'Sullivan, Charles A. Dana Foundation; Shari Powell, R.N., Johns Hopkins University School of Medicine; George Rebok, Ph.D., Johns Hopkins University School of Medicine; Jeff Reed, Johns Hopkins University School of Medicine; Susan Romberg, R.N., University of Pennsylvania; Cary Savage, Ph.D., Harvard University School of Medicine; James Schmeidler, Ph.D., Mount Sinai Medical Center; Laurie Stricks, Columbia University College of Physicians and Surgeons; Paula Ventura, Columbia University College of Physicians and Surgeons; Kelly Ware, Mount Sinai Medical Center; Naomi Zubin, Columbia University College of Physicians and Surgeons.

\section{References}

Anschutz, L., Camp, C., Markley, R., Kramer, J., 1987. Remembering mnemonics: a three-year follow-up on the effects of mnemonics training in elderly adults. Experimental Aging Research 13, 141-143.

Bandura, A., 1977. Self-efficacy: toward a unifying theory of behavioral change. Psychological Review 84, 191-215.

Crum, R.M., Anthony, J.C., Bassett, S.S., Folstein, M.F., 1993. Population-based norms for the Mini Mental State Examination by age and educational level. Journal of the American Medical Association 269, 2386-2391.

Delis, D.C., Kramer, J.H., Kaplan, E., Ober, B.A., 1986. California Verbal Learning Test - Adult Version. The Psychological Corporation, New York.

Delis, D.C., McKee, R., Massman, P.J., Kramer, J.H., Kaplan, E., Gettman, D., 1991. Alternate form of the California Verbal Learning Test: development and reliability. The Clinical Neuropsychologist 5, 154-163.

Dittmann-Kohli, F., Lachman, M., Kliegl, R., Baltes, P., 1991. Effects of cognitive training and testing on intellectual efficacy beliefs in elderly adults. Psychology and Aging 5, 215-227.

Folstein, M.F., Folstein, S.E., McHugh, P.R., 1975. Mini-Mental State: a practical method of grading the cognitive state of patients for the clinician. Journal of Psychiatric Research 12, 189-198.

Gilewski, M.J., Zelinski, E.M., Schaie, K.W., 1990. The Memory Functioning Questionnaire for the assessment of memory complaints in adulthood and old age. Psychology and Aging 5, 482-490.

Glosser, G., Goodglass, H., Biber, C., 1989. Assessing visual memory disorders. Psychological Assessment: A Journal of Consulting and Clinical Psychology 1, 82-91.

Jacobson, E., 1938. Progressive Relaxation. University of Chicago Press, Chicago.

Kaplan, E., Goodglass, H., Weintraub, S., 1983. The Boston Naming Test. Lea and Febiger, Philadelphia.
Kliegl, R., Smith, J., Baltes, P.B., 1989. Testing-the-limits and the study of adult age differences in cognitive plasticity of a mnemonic skill. Developmental Psychology 25, 247-256.

Kliegl, R., Smith, J., Baltes, P.B., 1990. On the locus and process of magnification of age differences during mnemonic training. Developmental Psychology 26, 894-904.

Lachman, M.E., Bandura, M., Weaver, S.L., Elliott, E., 1995. Assessing memory control beliefs: the Memory Controllability Inventory. Aging and Cognition 2, 67-84.

Lachman, M.E., Steinberg, E.S., Trotter, S.D., 1987. Effects of control beliefs and attributions on memory self-assessments and performance. Psychology and Aging 2, 266-271.

Lachman, M.E., Weaver, S., Bandura, M., Elliott, E., Lewkowicz, C., 1992. Improving memory and control beliefs through cognitive restructuring and self-generated strategies. Journal of Gerontology: Psychological Sciences 47, 293-299.

Morris, J.C., Heyman, A., Mohs, R.C., Hughes, J., van Balle, G., Fillenbaum, G., Mellits, E.D., Clark, C., and the CERAD investigators, 1989. The Consortium to Establish a Registry for Alzheimer's Disease (CERAD). Part 1. Clinical and neuropsychological assessment of Alzheimer's disease. Neurology 39, 1159-1165.

Poon, L.W., Walsh-Sweeney, L., Fozard, J.L., 1980. Memory skill training for the elderly: salient issues on the use of imagery mnemonics. In: Poon, L.W., Fozard, J.J., Cermak, L.S., Arenberg, D., Thompson, L.W. (Eds.), New Directions in Memory and Aging: Proceedings of the George A. Talland Memorial Conference. Lawrence Erlbaum, Hillsdale, NJ, pp. 461-484

Rebok, G.W., Balcerak, L.J., 1989. Memory self-efficacy and performance differences in young and old adults: the effect of mnemonic training. Developmental Psychology 25, 714-721.

Rosen, W.G., 1980. Verbal fluency in aging and dementia. Journal of Clinical Neuropsychology 2, 135-146.

Satz, P., Mogel, S., 1953. An abbreviation of the WAIS for clinical use. Clinical Psychology 18, 77-79.

Schaeffer, G., Poon, L.W., 1982. Individual variability in memory training with the elderly. Educational Gerontology 8, 217-229.

Schmitt, F.A., Murphy, M.D., Sanders, R.E., 1981. Training older adults free recall rehearsal strategies. Journal of Gerontology 36, 329-337.

Scogin, F., Bienias, J., 1988. A three-year follow-up of older adult participants in a memory skills training program. Psychology and Aging 3, 334-337.

Scogin, F., Storandt, M., Lott, L., 1985. Memory-skills training, memory complaints and depression in older adults. Journal of Gerontology 40, 562-568.

Sheikh, J.I., Hill, R.D., Yesavage, J.A., 1986. Long-term efficacy of cognitive training for age-associated memory impairment: a six-month follow-up study. Developmental Neuropsychology 2, 413-421.

Spielberger, C., 1971. Profile of Mood Scale (POMS). Educational and Industrial Testing Service, San Diego, CA. 
Stigsdotter-Neely, A., Backman, L., 1993a. Long-term maintenance of gains from memory training in older adults: two 3 1/2-year follow-up studies. Journal of Gerontology: Psychological Sciences 48, 233-237.

Stigsdotter-Neely, A., Backman, L., 1993b. Maintenance of gains following multifactorial and unifactorial memory training in late adulthood. Educational Gerontology 19, 105-117.

Stigsdotter-Neely, A., Backman, L., 1995. Effects of multifactorial memory training in old age: generalizability across tasks and individuals. Journal of Gerontology: Psychological Sciences 50B, 134-140.

Verhaeghen, P., Marcoen, A., Goossens, L., 1992. Improving memory performance in the aged through mnemonic training: a meta-analytic study. Psychology and Aging 7, 242-251.

Wechsler, D., 1981. Manual for the Wechsler Adult Intelli- gence Scale-Revised (WAIS-R). Psychological Corporation, New York.

West, R.L., Boatwright, L.K., Schleser, R., 1984. The link between memory performance, self-assessment and affective status. Experimental Aging Research 10, 197-200.

Willis, S., Schaie, K.W., 1986. Training the elderly on the ability factors of spatial orientation and inductive reasoning. Psychology and Aging 1, 239-247.

Yesavage, J.A., 1983. Imagery pretraining and memory training in the elderly. Gerontology 29, 271-275.

Yesavage, J.A., 1984. Relaxation and memory training in 39 elderly patients. American Journal of Psychiatry 141, 778-781.

Yesavage, J.A., Rose, T.L., Bower, G.H., 1983. Interactive imagery and affective judgments improve face-name learning in the elderly. Journal of Gerontology 38, 197-203. 